ARTÍCULOS 



\title{
EFECTOS DE LA CRISIS ECONÓMICA EN LA MOVILIDAD COTIDIANA DE LA REGIÓN METROPOLITANA DE BARCELONA
}

\author{
Oriol Marquet \\ Carme Miralles-Guasch" \\ *Center for Geospatial Analytics. North Carolina State University \\ oriol_marquet@ncsu.edu \\ " Departamento de Geografía. Universidad Autónoma de Barcelona \\ carme.miralles@uab.cat
}

\section{RESUMEN}

La crisis económica internacional ha tenido impactos en múltiples aspectos de la vida cotidiana en España. Como soporte indispensable de la vida cotidiana, la movilidad también se ha visto afectada por la pérdida de puestos de trabajo y la caída de la capacidad de gasto de las familias. La dimensión de los cambios sin embargo depende en gran parte de las condiciones inherentes del territorio, que tiene la capacidad de potenciar o restringir las estrategias de adaptación a la crisis. El artículo propone un análisis de la movilidad cotidiana en la Región Metropolitana de Barcelona para el periodo 2004-2012, examinando la evolución de los patrones de movilidad en los años previos y posteriores a la llegada de la crisis a España. Los impactos sobre la movilidad diaria se contextualizan en base a la resiliencia territorial de cada subámbito metropolitano: Barcelona, su $1^{\mathrm{a}}$ y $2^{\mathrm{a}}$ coronas metropolitanas, analizando las distintas posibilidades de adaptación que cada territorio ofrece a sus ciudadanos.

Palabras clave: movilidad; crisis económica; adaptación; Barcelona; resiliencia.

\section{ABSTRACT}

The current international crisis has had impacts on multiple aspects of everyday life in Spain. As an indispensable support of daily life, mobility has also been affected by the loss of jobs and the fall of spending power of families. The dimension of the changes, however, depends largely on the inherent conditions of the territory, which has the ability to

Fecha de recepción: noviembre 2015.

Fecha de aceptación: octubre 2016. 
enhance or constrain adaptation strategies to the crisis. The article analyzes daily mobility in the Barcelona Metropolitan Region for the period 2004-2012, examining the evolution of mobility patterns in the years before and after the arrival of the crisis in Spain. The impacts on daily mobility are contextualized upon the territorial resilience of each metropolitan area: Barcelona, the 1st and 2nd metropolitan crowns, also analyzing the different possibilities of adaptation that each area offers its citizens.

Keywords: mobility; economic crisis; adaptation strategies; Barcelona; resilience.

\section{LA CRISIS Y LAS CIUDADES ESPAÑOLAS}

La crisis financiera internacional, que surgió en 2008 y que aún no hemos superado, ha tenido efectos profundos en casi todos los aspectos de la sociedad española. La crisis, que surgió en el corazón de las principales economías mundiales y se extendió rápidamente gracias a las redes financieras globales, afectó de forma más intensa a economías periféricas como España, Grecia o Irlanda (Hadjimichalis, 2011). España, con una estructura económica vulnerable y excesivamente dependiente del sector de la construcción, ha sufrido de pleno sus impactos y consecuencias que se han extendido mucho más allá del sector financiero, con graves costes sociales. La recesión, afectó a la viabilidad de numerosas empresas, a través de la restricción del crédito. Ello condujo a la destrucción de puestos de trabajo y al aumento de la población desempleada. Este proceso, sumado al fin de la burbuja inmobiliaria, ha reducido el consumo y los ingresos de las administraciones públicas, cuya respuesta en forma de políticas de austeridad termina por acentuar los efectos de la crisis sobre la calidad de vida de los ciudadanos (Méndez, 2014).

Sin embargo, y a pesar de la magnitud de la crisis, no sólo en términos económicos, sino también sociales, existen aún pocos estudios sobre los efectos de la crisis en la vida cotidiana (Li y DaCosta, 2013). Para el caso específico de España, están los análisis de las consecuencias de la crisis en el impacto medioambiental del sector transporte (Mendiluce, Pérez-Arriaga, y Ocaña, 2010; Sobrino y Monzon, 2014; Zafrilla, López, Cadarso, y Dejuán, 2012), análisis generales de las secuelas en las ciudades españolas (Méndez y Prada-Trigo, 2014; Nel·lo y Donat, 2014; Trullén y Galletto, 2011) o análisis de los impactos en aspectos parciales de la movilidad cotidiana (Pitarch y Cano Maestro, 2012).

Uno de los indicadores que mejor miden la magnitud de las consecuencias de la crisis sobre la vida cotidiana, son los desplazamientos de la población, en la medida que reflejan las actividades que realizan los ciudadanos y los territorios que se utilizan (FreudendalPedersen, 2009). Sin embargo, aún no hay suficientes estudios sobre como la crisis ha modificado los hábitos de movilidad cotidiana de la población, como un eco de los profundos cambios sociales y económicos. En consecuencia, tampoco existe un análisis de las estrategias de adaptación que ha tenido que hacer la población, en lo referente a sus desplazamientos cotidianos, para amoldarse a una nueva realidad, caracterizada por la austeridad y la caída de los recursos económicos de las familias.

La crisis ha provocado, a nivel internacional, cambios similares en los hábitos de la población, no solo cuantitativos -menor número de desplazamientos, menores volúmenes de tráfico rodado- sino también cualitativos - cambios en la relación distancia viajada-modo 
de transporte, mayor peso de la movilidad personal, replanteamiento de viejos hábitos de movilidad-. Unos y otros muestran la profundidad de los cambios vitales que ha sufrido la población en muy pocos años. Mientras que el menor número de desplazamientos al trabajo puede ser una circunstancia coyuntural, temporal o transitoria. Los cambios en el carácter de la movilidad pueden ser más permanentes, puesto que afectan a los hábitos, costumbres y opiniones que nos formamos sobre la movilidad (Garcia-Sierra, van den Bergh, y MirallesGuasch, 2015), aspectos estructurales que pueden haber llegado para quedarse.

Es necesario, también, analizar los efectos desiguales de la crisis en términos sociales y territoriales. Por un lado, sus consecuencias no se han repartido, ni mucho menos, de forma uniforme entre todos los grupos sociales: al contrario, los grupos más vulnerables, también, son los más afectados por el aumento del desempleo y por la polarización de las desventajas relativas al acceso a los servicios básicos, entre ellos los sistemas de transporte. Un acceso que es fundamental para las grupos en riesgo de exclusión social (Cebollada i Frontera y Miralles-Guasch, 2006; Cumming, 2011, p. 190).

Por otro lado, la desigualdad social se ubica también en territorios diferentes. Una característica esencial de la crisis actual es que sus efectos han sido territorialmente heterogéneos (Hadjimichalis, 2011; Hassink, 2010). Tal y como indica Ricardo Méndez (2012), la perspectiva geográfica es indispensable para analizar los impactos desiguales de la crisis a nivel local y, en consecuencia, para diseñar políticas anticrisis adecuadas a las necesidades de cada área geográfica. Analizar la distribución geográfica de la crisis es también estudiar la distribución de la población vulnerable y la naturaleza resiliente de cada territorio. Y es que, los impactos de una crisis -económica o de otra índole- no solo dependen de su propia dimensión o de la vulnerabilidad de su población, sino también de las condiciones del territorio en que se produce. Las características de la ciudad, o territorio metropolitano, pueden aumentar o disminuir la posibilidad de adaptación de la población, por lo que la capacidad de respuesta de los ciudadanos a los efectos de la crisis es distinta según cada territorio.

El presente estudio propone un análisis detallado de la evolución de la movilidad, desde 2004 hasta 2012, en los distintos ámbitos territoriales de la Región Metropolitana de Barcelona. Utilizando la serie de encuestas de movilidad en día laborable (EMEF), se analiza cómo han cambiado las pautas de los desplazamientos cotidianos por los efectos de la crisis, comparando la etapa de crecimiento (2004-2007) con la etapa de recesión (2008-2012). También se diferencia entre la movilidad ocupacional (por motivo de trabajo o estudio) y la movilidad personal (otros motivos), así como las tendencias de cambio que se detectan en las dos coronas metropolitanas que conforman la RMB, además del municipio de Barcelona. Territorios que tienen características socioeconómicas distintas y particularidades morfológicas, lo que permite detectar dónde los hábitos cotidianos se han visto más afectados, a través de sus desplazamientos cotidianos, y visualizar como, las particularidades de los distintos territorios han incidido en la capacidad de ajuste a los cambios, configurando distintas estrategias de adaptación a la crisis

\section{LA CRISIS Y LA MOVILIDAD URBANA}

Las profundidades de las crisis, sus consecuencias y reacciones, se inscriben en territorios concretos y son cualitativamente diversas puesto que, cada actor reacciona de forma 
diferente ante un mismo estímulo. Tal y como explica Bertolini (2007), las respuestas a la crisis no son lineales sino complejas, con algunos cambios que dependen de las condiciones existentes (path-dependant) y otros que son totalmente impredecibles. En la actual crisis económica, la congelación de la inversión pública y la reducción de la capacidad de gasto de las familias han disminuido, entre otras consecuencias, el potencial de uso, por parte de ciertos grupos de población, de los transportes mecánicos. Al mismo tiempo, sin embargo, las políticas de austeridad se han inclinado, para disminuir costes, a reconcentrar servicios, lo que ha aumentado las distancias de los desplazamientos y, con ello, las necesidades de uso de los transportes mecánicos (Camarero, Cruz y Oliva, 2014). Esta doble dinámica lleva a Gomes et al., (2015) a considerar que en etapas de crisis económica, la exclusión por motivo de transporte es más frecuente y que por tanto también es más importante que nunca planificar servicios públicos y de transporte más socialmente sostenibles, accesibles y equitativos.

Los efectos de la crisis económica además se hacen más evidentes por producirse justo después de una etapa económicamente expansiva. En periodos de crecimiento económico como el que protagonizó España entre 1990-2007, la motorización y las distancias viajadas tienden a aumentar (Nielsen, 2015). Así, Sobrino et al. (2014) documentan para el caso español una primera etapa 1990-1997 con un gran crecimiento del parque automovilístico, y una segunda etapa anterior a la crisis (2002-2007) donde las condiciones de bonanza económica permitieron aumentar enormemente la cilindrada de los vehículos en circulación. Esta alza de la capacidad de acceso al vehículo privado, junto con la burbuja inmobiliaria y el incremento de los cambios de residencia hacia áreas urbanas periféricas, creó una estructura urbana dispersa y monofuncional que, terminó por aumentar la dependencia del automóvil (Bayona i Carrasco y Pujadas, 2014). En el caso concreto de la Región Metropolitana de Barcelona, Pujadas (2009) demuestra que entre 1980 y 2007 esta expansión metropolitana se produjo sin tener en cuenta la relación entre residencia y lugar de trabajo, lo que contribuye a explicar el aumento de las distancias viajadas, especialmente en el caso de la movilidad ocupacional.

A nivel internacional, se ha observado como la crisis ha hecho disminuir el uso de los transportes mecánicos: el transporte público debido a un empeoramiento del servicio derivado de las políticas de austeridad, y el transporte privado por la combinación de menos capacidad de gasto y un aumento de los precios del combustible (Christoforou y Karlaftis, 2011). Asimismo algunos estudios han encontrado que la crisis ha racionalizado la movilidad y las distancias viajadas, con un esfuerzo por parte de la población de aumentar la eficiencia de los viajes, eliminando desplazamientos sobrantes o excesivamente costosos en términos monetarios o de tiempo (Nielsen, 2015). En todos los casos, sin embargo, el elemento común es el descenso en el uso del vehículo privado, tanto en su peso relativo en el reparto modal como en las distancias viajadas. La relación entre crisis y descenso del uso del automóvil parece pues probada, aunque algunos autores como Metz (2013) o Millard-Ball y Schipper (2011) apuntan que parte del descenso puede también deberse a haber llegado al peak-travel, a la saturación de la demanda de viajes en vehículo privado justo antes del estallido de la crisis. Resta por explorar sin embargo, hasta qué punto esta reducción del uso del coche se ha podido llevar a cabo por igual en todas las áreas urbanas así como su impacto en términos de calidad de vida de los ciudadanos. 


\section{LAS ESTRATEGIAS DE RESILIENCIA EN LAS CIUDADES EN CRISIS}

La magnitud de los impactos de la crisis ha variado no solamente por la distribución desigual de los grupos de población más vulnerables por el territorio sino por las condiciones preexistentes y las características morfológicas de cada región. El concepto de resiliencia es de utilidad para entender como determinadas zonas ofrecen mayores posibilidades de adaptación a sus ciudadanos que otras, o lo que es lo mismo, que algunas áreas hacen posible cambiar más intensamente las formas de la movilidad sin perder su función final de sostener nuestra vida cotidiana.

El concepto de resiliencia, que proviene del ámbito medioambiental es ampliamente utilizado actualmente en otros ámbitos para describir la resistencia de un sistema frente a un conjunto de cambios y presiones externas (Jabareen, 2013). De las múltiples acepciones disponibles, la más adecuada para la escala urbana es la que entiende la resiliencia como la habilidad de un sistema de adaptarse ante un shock o una perturbación cambiando su forma si es necesario pero manteniendo su funcionalidad (Martin, 2012). La naturaleza de estas presiones externas sobre el sistema puede ser ecológica, financiera, social o cultural (Kärrholm, Nylund, y Prieto de la Fuente, 2014) y de la misma forma, los cambios pueden ser consecuencia de un shock puntual (desastres naturales) o un proceso de cambio prolongado en el tiempo (crisis económicas) (Pike, Dawley y Tomaney, 2010). En cualquier caso el concepto de resiliencia es indispensable para entender porque algunas regiones consiguen adaptarse, cambiar y renovarse ante una crisis, y otras regiones se muestran incapaces de hacerlo (Hassink, 2010).

En el caso de la RMB, no todos los ámbitos territoriales han sufrido las consecuencias de la crisis de la misma forma (Trullén y Galletto, 2011). Las características morfológicas y socioeconómicas de cada corona metropolitana han modulado el volumen de los impactos a la vez que han determinado la capacidad de adaptación de la población. En materia de transporte, un sistema resiliente es aquel que permite a los usuarios seguir con su movilidad cotidiana aún en el contexto de cambios inesperados. Eso incluye tanto transformaciones del propio sistema de transporte, como de los niveles socioeconómicos de los propios usuarios (Bertolini, 2007). El objetivo de un sistema de transporte resiliente no es volver al estado anterior a la crisis sino el de absorber los cambios sin perder la funcionalidad última del sistema de transporte que es garantizar la accesibilidad (Grieco, 2015). Para Reggiani et al., (2015) es precisamente la pérdida de nivel de accesibilidad lo que define a las poblaciones y territorios más vulnerables ante una crisis, aquellos que al tener que cambiar su forma de movilidad, pierden la capacidad de continuar con su movilidad cotidiana.

El proceso de crisis provoca cambios intangibles a nivel individual, como el replanteamiento de los hábitos de movilidad, la reestructuración de los espacios de actividad y el cuestionamiento de las narrativas estructurales que guían nuestras percepciones sobre la movilidad y los medios de transporte (Freudendal-Pedersen, 2009, p. 33). La crisis es un incentivo para cambiar de hábitos y reexaminar las pautas de movilidad de cada uno, lo que conduce en numerosas ocasiones al establecimiento de nuevas costumbres y rutinas (Dargay, 2001; Reggiani et al., 2015). Este proceso termina por reflejarse en una serie de cambios tangibles como son las variaciones en el reparto modal, la reducción del número de vehículos en circulación o el motivo de los desplazamientos. 
El carácter y la dimensión de estos cambios sin embargo dependen en parte de las posibilidades del área urbana y del sistema de transporte existente. Así, Grieco (2015) destaca que las áreas caminables, diseñadas en torno a las pequeñas distancias y al peatón, ofrecen mayores posibilidades de adaptación a los usuarios. Vivir en áreas poco accesibles andando en cambio, supone menores posibilidades de adaptación y mayores gastos en transporte (Mattingly y Morrissey, 2014). En general las áreas monocéntricas, dependientes de un solo medio de transporte, de baja densidad y caracterizadas por la separación funcional urbana, demuestran ser poco adaptables (Pike et al., 2010), mientras que aquellas con altas densidades de población, mixticidad de usos y con distintas opciones modales, ofrecen mayor capacidad de adaptación.

En este contexto la crisis es a la vez una prueba de resiliencia para nuestros sistemas de transporte y áreas urbanas, una ocasión para observar los mecanismos de adaptación de los usuarios y una oportunidad para romper tendencias y cambiar cualitativamente las formas de movilidad hegemónicas en la ciudad. Nuestro objetivo no debe ser el de volver a un sistema de transporte pre-crisis que era altamente insostenible y dependiente del vehículo privado, sino analizar los procesos de cambio que los usuarios han adoptado para mantener sus niveles de movilidad asumiendo los cambios provocados por la crisis (Kärrholm et al., 2014; Pickett, Cadenasso, y Grove, 2004). Pero para ello debemos entender primero los procesos de adaptación que los distintos grupos sociales y los distintos territorios han adoptado para navegar los efectos de la crisis.

\section{METODOLOGÍA}

La Región Metropolitana de Barcelona concentraba, en 2010, un 67\% de la población catalana y un $11 \%$ de la española. Se trata de un territorio con 164 municipios, distribuidos en siete comarcas y con una población total de 5.023.635 habitantes, cuyas dinámicas funcionales se estructuran bajo una lógica metropolitana. La región se articula a partir del municipio de Barcelona, ocupa unos $3.000 \mathrm{~km}^{2}$, en un radio de unos 30-45 km, y se compone de una Primera Corona metropolitana, conurbada al núcleo central, y una Segunda, más alejada, estructurada a partir de diversas ciudades de tamaño medio (Miralles-Guasch y Tulla Pujol, 2012).

La ciudad central, Barcelona, con 1.602.386 habitantes, ha colmatado su urbanización, tiene unos niveles de densidad de población altos y homogéneos $\left(15.802,6\right.$ habitantes $\left./ \mathrm{km}^{2}\right)$ y una buena distribución de servicios por sus distintos barrios. La primera corona de municipios alrededor de Barcelona se caracteriza por densidades desiguales, fruto de unos niveles de urbanización muy dispares (Miralles-Guasch y Donat, 2007). Por un lado se ubican tres de las grandes ciudades catalanas (Hospitalet de Llobregat, Badalona y Cornellà de Llobregat), con densidades mayores que Barcelona, por el otro existen zonas no urbanizadas e incluso rurales (Garcia-Sierra y van den Bergh, 2014). Por lo que en conjunto, la Corona, tiene una densidad bruta de población de $2.291 \mathrm{hab} / \mathrm{km}^{2}$ (Tabla 1).

La Segunda Corona, con 2.196.010 habitantes, se estructura a partir de siete ciudades históricas: Mataró, Granollers, Sabadell, Terrassa, Martorell, Vilafranca del Penedès y Vilanova i la Geltrú, en ellas vive el 25\% de la población de la Corona y generan dinámicas submetropolitanas a su alrededor (Marquet y Miralles-Guasch, 2013). El resto del territorio lo ocupan municipios de pequeño tamaño y tejido disperso, (con densidades por debajo de los 1000 hab $/ \mathrm{km}^{2}$ ), que suman 700.000 habitantes y han concentrado buena parte del crecimiento metropolitano en los últimos años (Bayona i Carrasco y Pujadas, 2014). 
Tabla 1

CARACTERÍSTICAS DE LA REGIÓN METROPOLITANA DE BARCELONA (2010)

\begin{tabular}{lccccc}
\hline $\begin{array}{c}\text { Ámbito } \\
\text { territorial }\end{array}$ & $\begin{array}{c}\text { Población } \\
\text { Total }\end{array}$ & $\begin{array}{c}\text { Superficie } \\
\mathbf{K m}^{2}\end{array}$ & $\begin{array}{c}\text { Densidad de } \\
\text { población } \\
\text { Hab/km }\end{array}$ & $\begin{array}{c}\text { RFDB } \\
\text { (miles } € \text { ) } \\
\text { (suma) }\end{array}$ & $\begin{array}{c}\text { INDEX } \\
\text { CAT100 } \\
\text { (base 2008) } \\
\text { (media) }\end{array}$ \\
\hline RMB & 5.023 .635 & $3.241,5$ & $1.549,7$ & $81.724 .452^{*}$ & $109,6^{*}$ \\
\hline Barcelona & 1.602 .386 & 101,4 & $15.802,6$ & 31.038 .101 & 114,5 \\
\hline $1^{\text {a } \text { corona }}$ & 1.225 .239 & 534,6 & 2.291 & 17.914 .034 & 95,1 \\
\hline $2^{\text {a corona }}$ & 2.196 .010 & $2.605,5$ & 842,8 & $32.772 .317^{*}$ & $99,3^{*}$ \\
\hline
\end{tabular}

Fuente: elaboración propia a partir de datos de Idescat.

*Sólo municipios de más de 5.000 habitantes.

De los tres ámbitos metropolitanos, la ciudad de Barcelona es la que cuenta con una renta media más elevada, un $14.5 \%$ por encima de la media catalana. La segunda corona se sitúa muy cerca de la media catalana, mientras que la primera corona es la que presenta valores más bajos, 5 puntos porcentuales, por debajo. Si entre 1985 y 2006, habían disminuido las diferencias en la distribución de renta entre territorios, la crisis ha alterado esta tendencia, aumentando las desigualdades, hasta volver a los niveles de 1990 (Trullén, 2014).

Para el análisis de la movilidad se han utilizado los datos de las Encuestas de Movilidad en Día Laborable (EMEF), correspondientes a los años 2004, 2007, 2010 y 2012, elaboradas por el IERMB. La encuesta busca conocer los hábitos de movilidad de la población mayor de 16 años residente en la Región Metropolitana de Barcelona. La información, recogida a través de metodología CATI, hace referencia a los desplazamientos de la persona entrevistada realizados el día laborable anterior a la entrevista e incluye variables relativas al desplazamiento así como características socioeconómicas de la persona entrevistada. El tamaño de muestra de las ediciones de la encuesta varía entre un mínimo de 4.642 entrevistados en 2004 y un máximo de 6.462 en 2012, en cualquier caso, muestra suficiente para analizar de forma desagregada la movilidad por motivos ocupacionales y personales. Al mismo tiempo, también permite distinguir entre aquellos que residen en el municipio de Barcelona, en el resto de la primera corona y en la segunda corona metropolitana.

\section{LA MOVILIDAD COTIDIANA ANTES Y DURANTE DE LA CRISIS (2004-2012)}

\section{V.1. Desplazamientos per cápita: un aumento sostenido}

La evolución reciente de la movilidad en la Región Metropolitana de Barcelona se ha caracterizado por un aumento en el número de desplazamientos per cápita. Esta misma dinámica se reproduce en la mayoría de países desarrollados y se relaciona con un aumento generalizado de la movilidad, tanto en quilómetros viajados como en frecuencia de viajes por individuo y día (Metz, 2012). Este aumento generalizado de la movilidad es una característica común de nuestra sociedad hipermóvil, que busca acometer cada vez más actividades en 
una misma unidad de tiempo. Tal y como describiera Urry (2000), la expansión de las TIC no ha disminuido nuestra necesidad de movilidad sino que al contrario la ha alimentado y la ha convertido en un requisito para participar de lleno en nuestra sociedad.

Desplazarnos equivale a acceder a los servicios y actividades que conforman el conjunto de nuestra actividad cotidiana: ir a trabajar, a comprar, al dentista o al teatro, toda actividad requiere de un desplazamiento. El aumento de los viajes por persona y día es pues un reflejo del constante deseo de aumentar el número de actividades que realizamos y a los servicios a los que tenemos acceso. Y a pesar que las externalidades no siempre deseadas de esta hipermovilidad han llevado a algunos a hablar de un nivel excesivo de viajes (Horner y O'Kelly, 2007; Mokhtarian y Handy, 2008) la realidad es que el aumento de desplazamientos per cápita está íntimamente relacionado con la búsqueda de una mejor calidad de vida y no puede suscitar más que valoraciones positivas.

La RMB no ha sido ajena a estas dinámicas de aumento generalizado de la movilidad y en los últimos años ha registrado también incrementos en el número de viajes por persona y día. La evolución exacta del número de desplazamientos entre 2004 y 2012 en los distintos ámbitos territoriales de la RMB puede consultarse en la tabla 2 que, como se observa, presenta claramente una tendencia alcista entre 2004 y 2010, momento en que alcanza un máximo de 4.07 viajes por persona, para descender hasta los 4 viajes por persona, en 2012. A nivel desagregado por coronas, puede constatarse como, la primera y segunda corona siguen la tendencia del conjunto del territorio pasando de 3,68 a 3,95 y de 3,87 a 4,24 entre el 2007 y 2010, respectivamente, para bajar ligeramente en el año 2012 en ambos casos, hasta 3,82 i 4,09. Sin embargo, la ciudad de Barcelona no sigue esta la tendencia a la baja sino que aumenta los desplazamientos por persona a lo largo del periodo pasando de 3,93 a 4,01.

Tabla 2

DESPLAZAMIENTOS PER CÁPITA EN LA REGIÓN METROPOLITANA DE BARCELONA 2004-2012

\begin{tabular}{lccccc}
\hline & \multicolumn{2}{c}{ Etapa pre-crisis } & \multicolumn{2}{c}{ Etapa crisis } & Diferencias \\
\hline Ámbito territorial & $\mathbf{2 0 0 4}$ & $\mathbf{2 0 0 7}$ & $\mathbf{2 0 1 0}$ & $\mathbf{2 0 1 2}$ & $\mathbf{2 0 0 7 - 2 0 1 2}$ \\
\hline RMB & 3,55 & 3,75 & 4,07 & 4,00 & 0,24 \\
\hline Barcelona & 3,47 & 3,65 & 3,93 & 4,01 & 0,36 \\
\hline $1^{\text {a }}$ Corona & 3,48 & 3,68 & 3,95 & 3,82 & 0,14 \\
\hline $2^{\text {a } \text { Corona }}$ & 3,67 & 3,87 & 4,24 & 4,09 & 0,22 \\
\hline
\end{tabular}

Fuente: elaboración propia a partir de datos de EMEF (2004, 2007, 2010, 2012).

En el conjunto de la RMB, los efectos de la crisis económica no han provocado una reducción en el número de desplazamientos por persona, pero sí una ralentización de su crecimiento. Así, entre el 2004 y el 2007 el número de viajes aumentó en un 0.07 anual, mientras que en el periodo posterior (2007-2012) el incremento se redujo situándose en el 0.05 desplazamientos adicionales por año. Pero esta dinámica es desigual por territorios. Mientras que Barcelona consigue mantener un ritmo de crecimiento del 0.07 anual entre 2007 y 2012, similar al que se observaba en el periodo anterior a la crisis, la primera y segunda corona metropolitana estancan su crecimiento. 
Dada la relación entre el número de desplazamientos y participación en actividades cotidianas, los resultados de la tabla 2 indican que, si bien los habitantes de Barcelona han seguido incrementando el número de actividades en las que participaban, los efectos de la crisis han provocado que los habitantes de la primera y segunda corona las hayan reducido.

\section{V.2. Motivo de desplazamiento: los desplazamientos ocupacionales bajan, los personales aumentan}

El impacto más evidente de la crisis económica ha sido, probablemente, la pérdida de puestos de trabajo. La destrucción de 359.000 empleos entre 2007 y 2012 en la región metropolitana (Nel·lo y Donat, 2014) ha supuesto una reestructuración profunda de las dinámicas de movilidad, que implica una modificación de la importancia de los motivos ocupacionales y personales en los desplazamientos totales (Tabla 3). Si en 2004, la movilidad ocupacional representaba un $43.2 \%$ del total en el área de estudio, en 2012, ya con un 22.6\% de población desocupada, representa solo el $28.2 \%$ de los desplazamientos. Este cambio no solo se debe a un descenso pronunciado de los viajes por este motivo, sino también a un ascenso en paralelo de los desplazamientos de tipo personal que ya puede detectarse en el periodo inmediatamente anterior a la crisis. Así, entre 2004 y 2007 los viajes de tipo ocupacional aumentaron en la RMB a un ritmo del 1,2\% anual, mientras que los motivos personales lo hicieron a un ritmo del 4,7\% anual (Tabla 4). A lo largo del periodo de la crisis (2007-2012), lo que se observa es una polarización de esta doble dinámica, con reducciones de la movilidad ocupacional del $-4.6 \%$ anuales acompañadas de aumentos del número de motivos personales a un ritmo del 7,2\% anual, hasta establecer un balance, en 2012, donde existen 2,5 desplazamientos personales por cada ocupacional (tabla 3).

Tabla 3

MOTIVO DEL DESPLAZAMIENTO, POR ÁMBITOS TERRITORIALES (\%)

\begin{tabular}{clccccc}
\hline & & \multicolumn{2}{c}{ Etapa pre-crisis } & \multicolumn{2}{c}{ Etapa crisis } & Diferencia \\
\hline $\begin{array}{c}\text { Ámbito } \\
\text { territorial }\end{array}$ & $\begin{array}{c}\text { Motivo del } \\
\text { desplazamiento }\end{array}$ & $\mathbf{2 0 0 4}$ & $\mathbf{2 0 0 7}$ & $\mathbf{2 0 1 0}$ & $\mathbf{2 0 1 2}$ & $\mathbf{2 0 0 7 - 2 0 1 2}$ \\
\hline \multirow{3}{*}{ RMB } & Ocupacional & $43,2 \%$ & $41,0 \%$ & $31,2 \%$ & $28,2 \%$ & $-15,0 \%$ \\
& Personal & $56,8 \%$ & $59,0 \%$ & $68,8 \%$ & $71,8 \%$ & $15,0 \%$ \\
& Total & 100 & 100 & 100 & 100 & - \\
\hline \multirow{3}{*}{ Barcelona } & Ocupacional & $40,8 \%$ & $41,0 \%$ & $32,2 \%$ & $29,8 \%$ & $-11,0 \%$ \\
& Personal & $59,2 \%$ & $59,0 \%$ & $67,8 \%$ & $70,2 \%$ & $11,0 \%$ \\
& Total & 100 & 100 & 100 & 100 & - \\
\hline \multirow{3}{*}{$1^{\text {a Corona }}$} & Ocupacional & $43,2 \%$ & $40,1 \%$ & $30,4 \%$ & $28,1 \%$ & $-15,1 \%$ \\
& Personal & $56,8 \%$ & $59,9 \%$ & $69,6 \%$ & $71,9 \%$ & $15,1 \%$ \\
& Total & 100 & 100 & 100 & 100 & - \\
\hline \multirow{3}{*}{$2^{\text {a Corona }}$} & Ocupacional & $45,4 \%$ & $41,5 \%$ & $30,8 \%$ & $27,1 \%$ & $-18,3 \%$ \\
& Personal & $54,6 \%$ & $58,5 \%$ & $69,2 \%$ & $72,9 \%$ & $18,3 \%$ \\
& Total & 100 & 100 & 100 & 100 & - \\
\hline
\end{tabular}

Fuente: elaboración propia a partir de datos de EMEF (2004, 2007, 2010, 2012). 
Una vez más, sin embargo, observamos que los cambios provocados por la crisis tienen un impacto territorialmente diverso. El municipio de Barcelona ha perdido una menor cantidad de puestos de trabajo, lo que provoca que su reducción de la movilidad ocupacional sea menor (-16.1\%) que la registrada en la Primera Corona (-22.9\%) o la Segunda Corona $(-28.1 \%)$. Destaca, sin embargo, que la movilidad personal, que tenía un crecimiento territorial desigual en la etapa 2004-2007, se homogeniza durante el periodo de crisis, con los tres ámbitos metropolitanos aumentando en más de un tercio su movilidad personal y con Barcelona aumentando sus desplazamientos en un 33 puntos porcentuales respecto a la etapa anterior.

Tabla 4

EVOLUCIÓN DEL NÚMERO DE DESPLAZAMIENTOS SEGÚN MOTIVO Y ÁMBITO TERRITORIAL

\begin{tabular}{|c|c|c|c|c|c|c|c|c|}
\hline \multirow[b]{3}{*}{$\begin{array}{l}\text { Ámbito } \\
\text { territorial }\end{array}$} & \multicolumn{4}{|c|}{$\begin{array}{c}\text { Etapa pre-crisis } \\
2004-2007\end{array}$} & \multicolumn{4}{|c|}{$\begin{array}{c}\text { Etapa crisis } \\
2007-2012\end{array}$} \\
\hline & \multicolumn{2}{|c|}{$\begin{array}{c}\text { Movilidad } \\
\text { Ocupacional }\end{array}$} & \multicolumn{2}{|c|}{ Movilidad Personal } & \multicolumn{2}{|c|}{$\begin{array}{c}\text { Movilidad } \\
\text { Ocupacional }\end{array}$} & \multicolumn{2}{|c|}{ Movilidad Personal } \\
\hline & $\begin{array}{c}\text { Diferencia } \\
\mathrm{N}^{\mathrm{o}} \text { viajes }\end{array}$ & $\begin{array}{c}\text { Diferencia } \\
\%\end{array}$ & $\begin{array}{l}\text { Diferencia } \\
\mathrm{N}^{\mathrm{o}} \text { viajes }\end{array}$ & $\begin{array}{c}\text { Diferencia } \\
\%\end{array}$ & $\begin{array}{l}\text { Diferencia } \\
\mathrm{N}^{0} \text { viajes }\end{array}$ & $\begin{array}{c}\text { Diferencia } \\
\%\end{array}$ & $\begin{array}{l}\text { Diferencia } \\
\mathrm{N}^{\circ} \text { viajes }\end{array}$ & $\begin{array}{c}\text { Diferencia } \\
\%\end{array}$ \\
\hline RMB & 117911 & $3,7 \%$ & 592120 & $14,0 \%$ & -766867 & $-22,9 \%$ & 1728482 & $35,9 \%$ \\
\hline Barcelona & 42681 & $4,1 \%$ & 61336 & $4,0 \%$ & -176475 & $-16,1 \%$ & 582277 & $36,9 \%$ \\
\hline $1^{\mathrm{a}}$ Corona & -1979 & $-0,2 \%$ & 135031 & $12,9 \%$ & -180561 & $-22,9 \%$ & 380499 & $32,2 \%$ \\
\hline $2^{\mathrm{a}}$ Corona & 77209 & $5,6 \%$ & 395753 & $23,8 \%$ & -409831 & $-28,1 \%$ & 765706 & $37,2 \%$ \\
\hline
\end{tabular}

Fuente: elaboración propia a partir de datos de EMEF (2004, 2007, 2010, 2012).

\section{V.3. Distribución modal de los desplazamientos: menos coches, más transportes activos}

Con la caída de la renta familiar disponible, el coste del desplazamiento en modos motorizados -ya sean públicos o privados- aumenta en términos relativos. La necesidad de ahorro y la búsqueda de una mayor eficiencia en el transporte han provocado numerosos cambios en el reparto modal (Tabla 5). En el global de la RMB, el periodo pre crisis 2004-2007, habían aumentado del $1 \%$ y del $1.4 \%$ en el uso de los medios no motorizado y privados, respectivamente. Con el estallido de la crisis, entre 2007 y 2012, se produce un trasvase desde el transporte motorizado hacia los no motorizados, cuyo peso en el reparto modal aumenta en 8 puntos porcentuales. Este mismo fenómeno se observa en todos los ámbitos territoriales de la RMB, pero con algunas características distintivas. Así, mientras en Barcelona el descenso de la movilidad motorizada se reparte entre transporte privado (-6.5) y transporte público (-1.9), en la primera y segunda coronas metropolitanas el aumento en el uso del caminar e ir en bici procede, exclusivamente, del descenso en el uso del vehículo privado, que llega a ser de 12 puntos porcentuales en la Segunda corona metropolitana. Es destacable el mantenimiento/aumento del uso del transporte público en ambas coronas, especialmente cuando, en el mismo periodo, el precio del billete ha subido un 34\%. Mientras que la morfología urbana 
compacta y la mixticidad de usos permiten en Barcelona concentrar los desplazamientos en el ámbito local y barrial, accesibles con medios no motorizados, la morfología dispersa, más presente en la Segunda corona metropolitana, obliga a que, parte del descenso del uso del vehículo privado tenga que ser asumido por el transporte público.

El impacto de la crisis lleva a que, en 2012, el uso de los medios no motorizados en la Segunda corona llegue a los niveles existentes en 2004 en la ciudad de Barcelona, y en el global de la RMB ya casi la mitad (46.3\%) de los desplazamientos que se realizan en un día laborable se realizan en un medio no motorizado.

Tabla 5

DISTRIBUCIÓN MODAL, POR ÁMBITOS TERRITORIALES (\%)

\begin{tabular}{llccccc}
\hline & & \multicolumn{2}{c}{ Etapa pre-crisis } & \multicolumn{2}{c}{ Etapa crisis } & Diferencia \\
\hline $\begin{array}{l}\text { Ámbito } \\
\text { territorial }\end{array}$ & Modo de transporte & $\mathbf{2 0 0 4}$ & $\mathbf{2 0 0 7}$ & $\mathbf{2 0 1 0}$ & $\mathbf{2 0 1 2}$ & $\mathbf{2 0 0 7 - 2 0 1 2}$ \\
\hline \multirow{3}{*}{ RMB } & Caminar + bici & 37,2 & 37,6 & 43,3 & 46,3 & $+8,7$ \\
& Transporte público & 20,6 & 19,6 & 19,5 & 19,0 & $-0,6$ \\
& Transporte privado & 42,2 & 42,8 & 37,3 & 34,7 & $-8,1$ \\
& Total & 100,0 & 100,0 & 100,0 & 100,0 & - \\
\hline \multirow{5}{*}{ Barcelona } & Caminar + bici & 38,5 & 40,5 & 46,1 & 48,9 & $+8,4$ \\
& Transporte público & 34,8 & 32,7 & 31,2 & 30,8 & $-1,9$ \\
& Transporte privado & 26,7 & 26,8 & 22,7 & 20,3 & $-6,5$ \\
& Total & 100,0 & 100,0 & 100,0 & 100,0 & - \\
\hline \multirow{5}{*}{$1^{\text {a Corona }}$} & Caminar + bici & 29,5 & 32,4 & 38,9 & 41,6 & $+9,2$ \\
& Transporte público & 19,0 & 18,1 & 18,5 & 18,2 & $+0,1$ \\
& Transporte privado & 51,5 & 49,5 & 42,6 & 40,1 & $-9,4$ \\
& Total & 100,0 & 100,0 & 100,0 & 100,0 & - \\
\hline \multirow{3}{*}{$2^{\text {a Corona }}$} & Caminar + bici & 29,3 & 27,2 & 34,7 & 38,2 & +11 \\
& Transporte público & 7,5 & 7,6 & 9,2 & 8,6 & +1 \\
& Transporte privado & 63,2 & 65,2 & 56,0 & 53,2 & -12 \\
& Total & 100,0 & 100,0 & 100,0 & 100,0 & - \\
\hline
\end{tabular}

Fuente: elaboración propia a partir de datos de EMEF (2004, 2007, 2010, 2012).

Este profundo cambio modal no se aplica sin embargo a todos los desplazamientos por igual, puesto que cada motivo de desplazamiento tiene sus propias características de viaje. En la tabla 6 se observa cómo, el comportamiento modal, no solo ha cambiado de forma distinta por territorios, sino que también se ha transformado en función del motivo. Los cambios modales se han efectuado en función de si el viaje era ocupacional o personal. En términos generales, la movilidad ocupacional, en 2012 en el conjunto de la RMB, sigue dominada por el uso de los transportes mecánicos, con un $45.7 \%$ en privado y un $32.3 \%$ en público. La movilidad personal, por su parte, se realiza en un $61.9 \%$ con modos no motorizados. Cabe destacar, en este punto, que incluso en la Segunda corona metropolitana, un 58\% de los viajes personales se realizan andando o en bicicleta. Por lo que, es el uso del transporte público 
(un $23.2 \%$ en $\mathrm{BCN}$ y $4.9 \%$ en la Segunda corona) el principal elemento diferenciador del reparto modal por motivos personales de Barcelona y las coronas metropolitanas.

Si se analizan los cambios acontecidos en el periodo de crisis, 2007-2012, también en la tabla 6, se observan dos tendencias distintas entre la movilidad ocupacional y la personal. Por lo que respecta a la primera, el descenso en el uso del vehículo privado (-7.3\%, RMB) es asumido, principalmente, por un aumento substancial del uso del transporte público $(+5.8 \%)$ y un aumento menor de los desplazamientos no motorizados $(+1.5 \%)$. El caso más claro de esta dinámica es el de la movilidad ocupacional en la ciudad de Barcelona, donde el descenso del $5.7 \%$ en el uso del vehículo privado es asumido, casi en su totalidad, por el transporte público, que aumenta un 5.3\%. Estos datos indican que se está produciendo un cambio modal sin una reconfiguración territorial de este tipo de desplazamientos. Es decir, la distancia entre residencia y lugar de trabajo es suficientemente grande como para necesitar de un medio motorizado, con lo que la mayoría de usuarios que modifican su comportamiento móvil lo hacen cambiando de un modo de transporte mecanizado más caro (coche) a otro medio de transporte mecanizado más asequible (transporte público).

Tabla 6

DISTRIBUCIÓN MODAL, POR ÁMBITOS TERRITORIALES (\%)

\begin{tabular}{|c|c|c|c|c|c|c|c|c|c|c|c|c|c|}
\hline \multirow[b]{2}{*}{ Ámbito } & \multirow[b]{2}{*}{$\begin{array}{l}\text { Modo de } \\
\text { transporte }\end{array}$} & \multicolumn{4}{|c|}{ Movilidad Ocupacional } & \multicolumn{4}{|c|}{ Movilidad Personal } & \multicolumn{2}{|c|}{$\begin{array}{c}\text { Evolución } \\
\text { movilidad } \\
\text { Ocupacional }\end{array}$} & \multicolumn{2}{|c|}{$\begin{array}{c}\text { Evolución } \\
\text { movilidad } \\
\text { Personal }\end{array}$} \\
\hline & & 2004 & 2007 & 2010 & 2012 & 2004 & 2007 & 2010 & 2012 & $\begin{array}{c}2004 \\
- \\
2007 \\
\end{array}$ & $\begin{array}{c}2007 \\
- \\
2012 \\
\end{array}$ & $\begin{array}{c}2004 \\
- \\
2007 \\
\end{array}$ & $\begin{array}{c}2007 \\
- \\
2012 \\
\end{array}$ \\
\hline \multirow{4}{*}{ RMB } & Andar+bici & 21,6 & 20,4 & 23,9 & 22,0 & 57,1 & 56,4 & 58,6 & 61,9 & $-1,1$ & 1,5 & $-0,7$ & 5,6 \\
\hline & T.Público & 27,0 & 26,5 & 29,3 & 32,3 & 14,7 & 14,8 & 13,6 & 12,9 & $-0,5$ & 5,8 & 0,1 & $-1,9$ \\
\hline & T.Privado & 51,4 & 53,1 & 46,8 & 45,7 & 28,3 & 28,9 & 27,8 & 25,2 & 1,7 & $-7,3$ & 0,6 & $-3,7$ \\
\hline & Total & 100 & 100 & 100 & 100 & 100 & 100 & 100 & 100 & 0 & 0 & 0 & 0 \\
\hline \multirow{4}{*}{$\mathrm{BCN}$} & Andar+bici & 21,8 & 25,3 & 27,5 & 25,6 & 58,0 & 57,3 & 60,8 & 64,4 & 3,5 & 0,3 & $-0,7$ & 7,1 \\
\hline & T.Público & 44,7 & 39,5 & 41,6 & 44,8 & 26,0 & 27,4 & 24,9 & 23,2 & $-5,3$ & 5,3 & 1,4 & $-4,2$ \\
\hline & T.Privado & 33,5 & 35,2 & 30,9 & 29,5 & 16,0 & 15,3 & 14,2 & 12,4 & 1,7 & $-5,7$ & $-0,7$ & $-2,9$ \\
\hline & Total & 100 & 100 & 100 & 100 & 100 & 100 & 100 & 100 & 0 & 0 & 0 & 0 \\
\hline \multirow{4}{*}{$1^{\text {a }}$ Corona } & Andar+bici & 20,6 & 18,1 & 20,4 & 20,1 & 63,0 & 63,1 & 64,2 & 65,3 & $-2,5$ & 2,0 & 0,1 & 2,2 \\
\hline & T.Público & 29,2 & 31,1 & 34,0 & 35,5 & 12,7 & 14,1 & 13,1 & 13,0 & 1,9 & 4,4 & 1,4 & $-1,1$ \\
\hline & T.Privado & 50,2 & 50,8 & 45,6 & 44,4 & 24,3 & 22,8 & 22,7 & 21,7 & 0,6 & $-6,4$ & $-1,5$ & $-1,1$ \\
\hline & Total & 100 & 100 & 100 & 100 & 100 & 100 & 100 & 100 & 0 & 0 & 0 & 0 \\
\hline \multirow{4}{*}{$2^{\text {a }}$ Corona } & Andar+bici & 22,0 & 18,1 & 23,0 & 19,9 & 52,5 & 51,7 & 54,0 & 58,2 & $-3,8$ & 1,7 & $-0,8$ & 6,5 \\
\hline & T.Público & 12,3 & 14,4 & 17,1 & 19,5 & 5,6 & 5,3 & 5,6 & 4,9 & 2,2 & 5,1 & $-0,3$ & $-0,5$ \\
\hline & T.Privado & 65,8 & 67,4 & 59,8 & 60,6 & 41,9 & 43,0 & 40,3 & 36,9 & 1,7 & $-6,8$ & 1,1 & $-6,1$ \\
\hline & Total & 100 & 100 & 100 & 100 & 100 & 100 & 100 & 100 & 0 & 0 & 0 & 0 \\
\hline
\end{tabular}

Fuente: elaboración propia a partir de datos de $\operatorname{EMEF}(2004,2007,2010,2012)$. 
La movilidad personal, por su parte, no sigue estos mismo patrones sino que en el periodo 2007-2012, tanto el transporte público como el privado descienden a favor de los desplazamientos a pie y en bicicleta $(+5.6 \%)$. En los cambios modales dentro de la movilidad personal, la configuración territorial juega también un papel importante, puesto que, si en Barcelona el descenso de la movilidad mecanizada se localiza, en mayor medida, en la pérdida de usuarios del transporte público (-4.2\%), en el caso de la Segunda corona metropolitana, esta pérdida se debe casi exclusivamente al descenso en el uso del vehículo privado $(-6.1 \%)$.

En cualquier caso, el común denominador del impacto de la crisis en la movilidad personal es un aumento de la movilidad no motorizada, lo que indica la aparición de dos factores complementarios. Por un lado, las distancias que anteriormente se consideraban demasiado largas para ser recorridas con medios no motorizados, ahora se consideran asequibles. En segundo lugar, la crisis ha provocado una reconfiguración territorial de nuestro espacio de actividad diaria, donde las actividades personales se ubican en distancias caminables, y por ende más cercanas.

\section{V.4. Menos distancia recorrida, más contención}

Esta reconfiguración territorial es especialmente interesante porque indica que los impactos de la crisis en el ámbito de la movilidad van más allá de una simple substitución de un medio de transporte por otro. La crisis impulsa a repensar el uso territorial que se hace de la región metropolitana. Si el periodo alcista se había caracterizado por un aumento de las distancias viajadas y una intensificación de las interrelaciones metropolitanas (Nel·lo y Donat, 2014), gracias, en gran parte, al dinamismo proporcionado por la movilidad en vehículo privado, el periodo de crisis revierte en parte esta dinámica de expansión espacial. Entre 2007 y 2012 la suma total de kilómetros recorridos en la Región Metropolitana descendió en un $-1.5 \%$, lo que indica un proceso de ralentización de la expansión metropolitana. Sin embargo, para apreciar cómo han cambiado las distancias viajadas se tiene que introducir la tipología de desplazamiento y el medio de transporte (Tabla 7).

Si bien ya se ha comentado como la movilidad ocupacional, en la RMB, ha descendido numéricamente, en el periodo 2007-2012 la tendencia no ha sido la de acortar los desplazamientos al trabajo, al contrario, ha continuado la tendencia pre-crisis de unos desplazamientos al trabajo cada vez más alejados (Pujadas, 2009). Así, en 2012, los viajes ocupacionales eran, de media, un $8 \%$ más largos que los de 2007, una diferencia mucho más pronunciada en la segunda corona $(+16 \%)$ que en Barcelona $(+1,3 \%)$ o en la primera corona $(+3,8 \%)$. Estos hechos coinciden plenamente con los descubiertos por Nielsen (2015) al estudiar los efectos de la crisis en el área metropolitana de Copenhague donde se detecta un aumento mayor de las distancias viajadas por motivo ocupacional en aquellas zonas con menor densidad de empleos. En Barcelona, el principal cambio en la movilidad ocupacional es que, las distancias que se hacen en coche son menores y en cambio las distancias en medios no motorizados aumentan en un $11 \%$. En la segunda corona metropolitana en cambio el aumento del $16 \%$ en las distancias al trabajo no solo se solventa con un aumento del alcance de la movilidad no motorizada sino que las distancias en transporte público y privado también aumentan un $7.1 \%$ y $9,2 \%$ respectivamente. 
Tabla 7

DISTANCIA MEDIA RECORRIDA (KM) POR DESPLAZAMIENTO, SEGÚN ÁMBITO TERRITORIAL, MODO DE TRANSPORTE Y MOTIVO DE VIAJE

\begin{tabular}{|c|c|c|c|c|c|c|c|c|c|}
\hline \multirow[b]{2}{*}{ Ámbito } & \multirow[b]{2}{*}{$\begin{array}{l}\text { Modo de } \\
\text { transporte }\end{array}$} & \multicolumn{4}{|c|}{ Movilidad Ocupacional } & \multicolumn{4}{|c|}{ Movilidad Personal } \\
\hline & & 2007 & 2010 & 2012 & 2007-2012 & 2007 & 2010 & 2012 & 2007-2012 \\
\hline \multirow{4}{*}{ RMB } & Andar+bici & 1,16 & 1,18 & 1,32 & $13,9 \%$ & 1,41 & 1,40 & 1,34 & $-5,6 \%$ \\
\hline & T.Público & 15,69 & 13,93 & 16,36 & $4,2 \%$ & 10,86 & 12,93 & 11,04 & $1,7 \%$ \\
\hline & T.Privado & 7,41 & 7,36 & 7,58 & $2,2 \%$ & 5,95 & 5,97 & 5,78 & $-2,9 \%$ \\
\hline & Total & 8,19 & 7,44 & 8,85 & $8,0 \%$ & 3,84 & 3,97 & 3,52 & $-8,4 \%$ \\
\hline \multirow{4}{*}{$\mathrm{BCN}$} & Andar+bici & 1,46 & 1,49 & 1,62 & $11,1 \%$ & 1,40 & 1,46 & 1,34 & $-4,5 \%$ \\
\hline & T.Público & 12,65 & 13,13 & 12,65 & $0,0 \%$ & 9,41 & 12,74 & 10,02 & $6,5 \%$ \\
\hline & T.Privado & 8,76 & 8,08 & 8,22 & $-6,2 \%$ & 8,15 & 7,38 & 8,00 & $-1,8 \%$ \\
\hline & Total & 8,18 & 7,88 & 8,28 & $1,3 \%$ & 4,14 & 4,75 & 3,87 & $-6,7 \%$ \\
\hline \multirow{4}{*}{$1^{\text {a }}$ Corona } & Andar+bici & 1,01 & 1,02 & 1,17 & $16,0 \%$ & 1,44 & 1,38 & 1,38 & $-4,0 \%$ \\
\hline & T.Público & 15,74 & 13,60 & 16,44 & $4,5 \%$ & 12,22 & 12,02 & 11,64 & $-4,7 \%$ \\
\hline & T.Privado & 7,70 & 6,91 & 7,34 & $-4,6 \%$ & 6,57 & 6,26 & 6,19 & $-5,8 \%$ \\
\hline & Total & 8,86 & 7,59 & 9,19 & $3,8 \%$ & 3,9 & 3,73 & 3,63 & $-6,9 \%$ \\
\hline \multirow{4}{*}{$2^{\mathrm{a}}$ Corona } & Andar+bici & 0,92 & 0,98 & 1,07 & $16,5 \%$ & 1,40 & 1,37 & 1,30 & $-7,3 \%$ \\
\hline & T.Público & 21,79 & 15,88 & 23,34 & $7,1 \%$ & 14,53 & 14,74 & 13,83 & $-4,8 \%$ \\
\hline & T.Privado & 6,78 & 7,26 & 7,40 & $9,2 \%$ & 5,14 & 5,51 & 5,08 & $-1,3 \%$ \\
\hline & Total & 7,85 & 7,03 & 9,15 & $16,6 \%$ & 3,57 & 3,55 & 3,19 & $-10,8 \%$ \\
\hline
\end{tabular}

Fuente: elaboración propia a partir de datos de EMEF $(2007,2010,2012)$.

Las tendencias que se detectan en la movilidad personal son totalmente distintas, puesto que como se puede observar en la parte derecha de la tabla 7, todas las distancias se reducen con la crisis. Las distancias viajadas en los motivos personales aparecen, casi, inversamente proporcionales con las distancias de la movilidad ocupacional, de tal forma que, a nivel global de la RMB, las distancias personales se reducen en un $8.4 \%$ en relación al aumento del $8 \%$ de las ocupacionales, y cuando observamos la misma relación por ámbitos territoriales se ve que, aquellos territorios que han aumentado más su movilidad ocupacional también reducen la personal en una mayor proporción. Pareciera así que, la movilidad ocupacional y personal son dos esferas conectadas, a modo de vasos comunicantes y que los ciudadanos están reconfigurando el alcance y la distribución de su movilidad personal en función a las necesidades de la movilidad ocupacional que tiene por sus características un carácter más fijo y menos adaptable. 


\section{CONCLUSIONES}

La movilidad en la Región Metropolitana de Barcelona ha experimentado intensos cambios en el periodo 2007-2012, como consecuencia de la crisis económica y sus repercusiones en la vida cotidiana de las personas. Existen, ante las crisis, tres estrategias posibles de adaptación de la movilidad. Por un lado, reducir el número total de actividades cotidianas, lo que implica disminuir las tareas cotidianas. Por otro, la adaptación puede significar cambios en el reparto modal, en función de su coste y accesibilidad. Por último, también se puede cambiar el uso de los territorios de la cotidianidad y con ello la escala territorial de cada individuo. Una cuarta opción sería la de aumentar los tiempos invertidos en desplazamientos, aunque tal y como demuestran Ajenjo Cosp y García Roman (2014) en España los tiempos de desplazamiento se han mantenido estables. En el caso de la Región Metropolitana de Barcelona, la crisis no ha reducido el número de desplazamientos por persona, por lo que, el proceso de adaptación se produce a través del cambio de los patrones de uso de los distintos modos de transporte y de la modificación de las distancias viajadas. En su mayoría, lo que se observa es una combinación de ambas estrategias, ya que optar por destinos más cercanos posibilita el trasvase modal desde los medios motorizados hacia los no-motorizados, sin que ello afecte a las actividades cotidianas.

Ante las exigencias de la crisis, los ciudadanos han tenido que replantearse sus estrategias de movilidad, racionalizando el uso de ciertos medios de transporte, lo que ha mantenido el número de desplazamientos que realizan en su día a día. Este cambio de tendencia que se produce en la transición desde el periodo de crecimiento 2004-2007 al periodo de crisis 2007-2012, demuestra que la demanda de transporte es flexible, pues depende de las circunstancias externas e internas de los ciudadanos.

La primera conclusión a la que se llega, tras analizar la evolución de la movilidad en el periodo 2007-2012, es que la crisis no ha reducido el volumen total de desplazamientos en la RMB. La caída de la movilidad ocupacional, provocada por la pérdida de lugares de trabajo, ha sido compensada e incluso superada por el aumento paralelo de la movilidad personal. Los ciudadanos reinvierten el ahorro de los costes monetarios o temporales de la pérdida de un desplazamiento ocupacional, en un mayor número de desplazamientos personales. Del mismo modo, el descenso en el uso del vehículo privado ha supuesto un aumento casi equivalente de los medios no motorizados. Lo que ha cambiado no ha sido el volumen de movilidad sino sus características cotidianas, su naturaleza. La crisis supone, entre otras cosas, eliminar gastos en los presupuestos familiares y ello ha incidido directamente en el uso del automóvil. Lo que indica que en la Región Metropolitana de Barcelona existía una sobre utilización del modo privado, pues parte de los viajes realizados en coche se podían hacer en otro medio de transporte.

Sin embargo, el proceso de adaptación de la movilidad a la crisis no ha sido homogéneo en toda el área metropolitana. Las condiciones territoriales de cada ámbito metropolitano potencian o no la capacidad de adaptación de sus habitantes (Marquet y Miralles-Guasch, 2017). Al igual que las economías diversificadas absorben mejor los impactos de los shocks financieros (Pike et al., 2010), también los sistemas de transporte diversos y los territorios compactos y mixtos, permiten absorber mejor las consecuencias de la crisis económica. 
Así, es posible distinguir estrategias de adaptación distintas para Barcelona, la primera y la segunda corona metropolitanas. Por una parte, el ajuste en Barcelona combina el cambio modal con una apuesta por los desplazamientos de proximidad que ya venía apuntándose desde periodos anteriores (Marquet y Miralles-Guasch, 2015; Miralles-Guasch y Marquet Sarda, 2013). Con una configuración territorial densa y mixta y con un modelo de movilidad diversificado (Marquet y Miralles-Guasch, 2016), la ciudad permite que, sus habitantes cambien sus formas de movilidad y al mismo tiempo sigan aumentando el número de desplazamientos por persona y día. En el caso de la primera y segunda coronas metropolitanas, en cambio, el ajuste se produce más intensamente en lo que se refiere al cambio modal, con un descenso de los desplazamientos en transporte público y privado y un ascenso de los no-motorizados. La morfología del territorio, sin embargo, no permite una reducción de las distancias igual en dimensión a la de Barcelona. Tanto en la primera como en la segunda corona metropolitana, las distancias al trabajo han aumentado durante el periodo de la crisis con lo que se ha concentrado aún más la movilidad de tipo personal. En ambos casos estos ajustes han provocado que el ritmo de crecimiento de los desplazamientos por persona se haya ralentizado con respecto al de Barcelona.

Los datos indican también que, a medida que nos alejamos del centro de la corona metropolitana, los ajustes que se han tenido que realizar para adaptarse a la crisis han sido más profundos. Son precisamente los territorios de la segunda corona metropolitana, nacidos en parte de la burbuja inmobiliaria, los que son menos resilientes, puesto que exigen mayores esfuerzos de adaptación a sus ciudadanos. La heterogeneidad territorial y morfológica de la segunda corona sin embargo pueden matizar estos resultados. El impacto real que estos cambios han tenido en la calidad de vida de los ciudadanos es difícil de cuantificar, puesto que es posible que los territorios que han exigido mayores cambios también sean habitados por perfiles de población con una mayor capacidad de adaptación. El grado final de exposición a los efectos de la crisis dependerá, no solo de la resiliencia del territorio sino también de la adaptabilidad de sus habitantes (Reggiani et al., 2015). En adelante será importante pues, tener en cuenta tanto la distribución espacial de los grupos más vulnerables como la resiliencia territorial de cada ámbito geográfico.

Cabe tener en cuenta también que al utilizar un rango temporal amplio algunos de los resultados apuntados por el estudio pueden depender también de cambios en la estructura sociodemográfica de los habitantes de la Región Metropolitana de Barcelona o de cambios en la política de transportes introducidos durante estos años. Mientras que cambios demográficos como el progresivo envejecimiento de la población o el aumento y posterior descenso de la población inmigrante pueden haber alterado las dinámicas de movilidad en el nivel macro, algunas obras en infraestructuras de transporte pueden haber modificado los patrones de movilidad de zonas específicas.

Los efectos de la crisis nos sirven para constatar que los territorios resilientes permiten seguir con el día a día cambiando hábitos de transporte a la vez que se demuestra que es posible mantener la movilidad cotidiana, con un modelo más sostenible que el existente en la etapa anterior a 2007. En este contexto el objetivo de las políticas públicas no debe ser en ningún caso volver a recuperar los patrones de movilidad pre-crisis sino estabilizar los cambios provocados por esta, y aumentar la resiliencia territorial para que los cambios no supongan un impacto negativo para la vida cotidiana. Tal y como lo expresa Bertolini (2007), 
el transporte y la movilidad se rigen por un pensamiento evolutivo en el cual no existe un estado natural al que volver. Hay que trabajar por tanto no para revertir dichos cambios sino para adaptarse con éxito a ellos.

El camino es pues diseñar las áreas urbanas pensando en la accesibilidad en modos no motorizados como el caminar o la bicicleta, transportes que proporcionan niveles igualitariamente altos de accesibilidad a todos los grupos de población (Marquet y Miralles-Guasch, 2014; Southworth, 2005). Diseñar la ciudad caminable como solución a la movilidad en tiempos de crisis implica fortalecer la escala local para aliviar la necesidad de movilidad de larga distancia, contribuyendo a aliviar también la presión sobre el sistema de transporte motorizado (Grieco, 2015). Además, y dado que el sistema y las políticas de transporte se caracterizan por tener una inercia larga y periodos de transición lentos, las estrategias en el corto y el largo plazo no son iguales ya que los desafíos de una y otra escala temporal son también de carácter distinto. En el corto plazo, debemos pensar en cómo garantizar la accesibilidad con la infraestructura y el sistema de transporte existente. Mientras que a largo plazo se debe plantear como rediseñamos el territorio y el transporte para construir un sistema más resiliente, que facilite las estrategias de adaptación y que sea inclusivo e igualitario.

\section{REFERENCIAS}

AJENJO COSP, M., y GARCÍA ROMÁN JOAN. (2014). Cambios en el uso del tiempo de las parejas ¿Estamos en el camino hacia una mayor igualdad? Revista Internacional de Sociología, 72(2), 453-476. http://doi.org/10.3989/ris.2012.05.28

BAYONA I CARRASCO, J., y PUJADAS, I. (2014). Movilidad residencial y redistribución de la población metropolitana: los casos de Madrid y Barcelona. EURE (Santiago), 40(119), 261-287.

BERTOLINI, L. (2007). Evolutionary urban transportation planning: An exploration. Environment and Planning A, 39(8), 1998-2019. http://doi.org/10.1068/a38350

CAMARERO, L., CRUZ, F., y OLIVA, J. (2014). Rural sustainability, inter-generational support and mobility. European Urban and Regional Studies. http://doi. org/10.1177/0969776414539338

CEBOLLADA I FRONTERA, À., y MIRALLES-GUASCH, C. (2006). Mobility and Urban Transport in Metropolitan Barcelona Accessibility versus Exclusion. In G. Arvastson y T. Butler (Eds.), Multiculturalism and Cities (pp. 19-30). Berlin: University of Copenhaguen.

CHRISTOFOROU, Z., y KARLAFTIS, M. (2011). Urban restructuring and transportation in the financial crisis era: A study of the Athens CBD. Association for European Transport Contributors.

CUMMING, G. (2011). Spatial Resilience in Social-Ecological Systems. London: Springer.

DARGAY, J. M. (2001). The effect of income on car ownership: evidence of asymmetry. Transportation Research Part A: Policy and Practice, 35(9), 807-821. http://doi. org/10.1016/S0965-8564(00)00018-5

FREUDENDAL-PEDERSEN, M. (2009). Mobility in daily life. Between freedom and Unfreedom. Farnham: Ashgate. 
GARCIA-SIERRA, M., y VAN DEN BERGH, J. C. J. M. (2014). Policy mix to reduce greenhouse gas emissions of commuting: A study for Barcelona, Spain. Travel Behaviour and Society, 1(3), 113-126. http://doi.org/10.1016/j.tbs.2014.06.001

GARCIA-SIERRA, M., VAN DEN BERGH, J. C. J. M., y MIRALLES-GUASCH, C. (2015). Behavioural economics, travel behaviour and environmental-transport policy. Transportation Research Part D: Transport and Environment, 41, 288-305. http://doi. org/10.1016/j.trd.2015.09.023

GOMES, R., PINHO DE SOUSA, J., y GALVÃO DIAS, T. (2015). Sustainable Demand Responsive Transportation systems in a context of austerity: The case of a Portuguese city. Research in Transportation Economics, 1-10. http://doi.org/10.1016/j.retrec.2015.07.011

GRIECO, M. (2015). Poverty mapping and sustainable transport: A neglected dimension. Research in Transportation Economics. http://doi.org/10.1016/j.retrec.2015.07.002

HADJIMICHALIS, C. (2011). Uneven geographical development and socio-spatial justice and solidarity: European regions after the 2009 financial crisis. European Urban and Regional Studies, 18(3), 254-274. http://doi.org/10.1177/0969776411404873

HASSINK, R. (2010). Regional resilience: A promising concept to explain differences in regional economic adaptability? Cambridge Journal of Regions, Economy and Society, 3(1), 45-58. http://doi.org/10.1093/cjres/rsp033

HORNER, M. W., y O'KELLY, M. E. (2007). Is non-work travel excessive? Journal of Transport Geography, 15(6), 411-416. http://doi.org/10.1016/j.jtrangeo.2006.12.003

JABAREEN, Y. (2013). Planning the resilient city: Concepts and strategies for coping with climate change and environmental risk. Cities, 31, 220-229. http://doi.org/10.1016/j. cities.2012.05.004

KÄRRHOLM, M., NYLUND, K., y PRIETO DE LA FUENTE, P. (2014). Spatial resilience and urban planning: Addressing the interdependence of urban retail areas. Cities, 36, 121-130. http://doi.org/10.1016/j.cities.2012.10.012

LI, Y., y DACOSTA, M. N. (2013). Transportation and income inequality in China: 19782007. Transportation Research Part A: Policy and Practice, 55, 56-71. http://doi. org/10.1016/j.tra.2013.08.006

MARQUET, O., y MIRALLES-GUASCH, C. (2013). Dinámicas y planeamiento territorial en los espacios metropolitanos periféricos: el municipio de Sabadell dentro del Área Metropolitana de Barcelona. In J. Noyola, C. De Mattos, y A. Orellana (Eds.), Urbanización en tiempos de crisis. Impactos desafios y propuestas (p. 623). Santiago de Chile: Instituto de Estudios Urbanos y Territoriales.

MARQUET, O., y MIRALLES-GUASCH, C. (2014). Walking short distances. The socioeconomic drivers for the use of proximity in everyday mobility in Barcelona. Transportation Research Part A: Policy and Practice, 70, 210-222. http://doi.org/http://dx.doi. org/10.1016/j.tra.2014.10.007

MARQUET, O., y MIRALLES-GUASCH, C. (2015). The Walkable city and the importance of the proximity environments for Barcelona's everyday mobility. Cities, 42, 258-266. http://doi.org/10.1016/j.cities.2014.10.012

MARQUET, O., y MIRALLES-GUASCH, C. (2016). City of Motorcycles. On how objective and subjective factors are behind the rise of two-wheeled mobility in Barcelona. Transport Policy, 52, 37-45. http://doi.org/10.1016/j.tranpol.2016.07.002 
MARQUET, O., y MIRALLES-GUASCH, C. (2017). Resilient territories and mobility adaptation strategies in times of economic recession. Evidence from the Metropolitan Region of Barcelona, Spain 2004-2012. European Urban and Regional Studies, 1-15. http://doi.org/http://dx.doi.org/10.1177\%2F0969776417703158

MARTIN, R. (2012). Regional economic resilience, hysteresis and recessionary shocks. Journal of Economic Geography, 12(1), 1-32. http://doi.org/10.1093/jeg/lbr019

MATTINGLY, K., y MORRISSEY, J. (2014). Housing and transport expenditure: Sociospatial indicators of affordability in Auckland. Cities, 38, 69-83. http://doi.org/10.1016/j. cities.2014.01.004

MÉNDEZ, R. (2012). Crisis Económica y Reconfiguraciones Territoriales. In V Jornadas de Geografía Económica AGE Univ. de Girona 2012 (pp. 37-73).

MÉNDEZ, R. (2014). Crisis Económica y reconfiguraciones territoriales. In J. M. Albertos Puebla y J. L. Sánchez Hernández (Eds.), Geografía de la crisis económica de España (pp. 17-38). Universitat de Valencia, Servei de Publicacions.

MÉNDEZ, R., y PRADA-TRIGO, J. (2014). Crisis desempleo y vulnerabilidad en Madrid. Scripta Nova, XVIII(474), 1-13.

MENDILUCE, M., PÉREZ-ARRIAGA, I., y OCAÑA, C. (2010). Comparison of the evolution of energy intensity in Spain and in the EU15. Why is Spain different? Energy Policy, 38(1), 639-645. http://doi.org/10.1016/j.enpol.2009.07.069

METZ, D. (2012). Demographic determinants of daily travel demand. Transport Policy, 21, 20-25. http://doi.org/10.1016/j.tranpol.2012.01.007

METZ, D. (2013). Peak Car and Beyond: The Fourth Era of Travel. Transport Reviews, 33(3), 255-270. http://doi.org/10.1080/01441647.2013.800615

MILLARD-BALL, A., y SCHIPPER, L. (2011). Are We Reaching Peak Travel? Trends in Passenger Transport in Eight Industrialized Countries. Transport Reviews, 31(3), 357378. http://doi.org/10.1080/01441647.2010.518291

MIRALLES-GUASCH, C., y DONAT, C. (2007). Análisi de l'oferta i la demanda de polígons d'activitat a Catalunya. Papers: Regió Metropolitana de Barcelona: Territori, Estratègies, Planejament, 45, 9-36.

MIRALLES-GUASCH, C., y MARQUET SARDA, O. (2013). Dinámicas de proximidad en ciudades multifuncionales. CyTET Ciudad Y Territorio, Estudios Territoriales, XLV(177), 503-512.

MIRALLES-GUASCH, C., y TULLA PUJOL, A. F. (2012). La región metropolitana de Barcelona. Dinámicas territoriales recientes. Boletín de la Asociación de Geógrafos Españoles, (58), 299-318. Retrieved from http://dialnet.unirioja.es/servlet/ articulo?codigo $=3886882$

MOKHTARIAN, P. L., y HANDY, S. (2008). No Particular Place to Go: An Empirical Analysis of Travel for the Sake of Travel. Environment and Behavior, 41(2), 233-257. http://doi.org/10.1177/0013916507310318

NEL·LO, O., y DONAT, C. (2014). Los efectos territoriales de la crisis económica en la región metropolitana de Barcelona. In J. M. Albertos y J. L. Sánchez-Hernández (Eds.), Geografía de la crisis económica de España (pp. 565-608). València: Universitat de Valencia, Servei de Publicacions. 
NIELSEN, T. A. S. (2015). Changes in transport behavior during the financial crisis. An analysis of urban form, location and transport behavior in the greater Copenhagen area 2006-2011. Research in Transportation Economics, 1-10. http://doi.org/10.1016/j. retrec.2015.07.003

PICKETT, S. T. A, CADENASSO, M. L., y GROVE, J. M. (2004). Resilient cities: Meaning, models, and metaphor for integrating the ecological, socio-economic, and planning realms. Landscape and Urban Planning, 69(4), 369-384. http://doi.org/10.1016/j.landurbplan.2003.10.035

PIKE, A., DAWLEY, S., y TOMANEY, J. (2010). Resilience, adaptation and adaptability. Cambridge Journal of Regions, Economy and Society, 3(1), 59-70. http://doi. org/10.1093/cjres/rsq001

PITARCH, M. D., y CANO MAESTRO, I. (2012). El impacto de la crisis en la accesibilidad a los espacios productivos: el caso del área metropolitana de Valencia. In V Jornadas de Geografía Económica AGE (pp. 132-148). Girona.

PUJADAS, I. (2009). Movilidad residencial y expansión urbana en la región metropolitana de Barcelona, 1982-2005. Scripta Nova, XIII(290), 1-13.

REGGIANI, A., NIJKAMP, P., y LANZI, D. (2015). Transport resilience and vulnerability: The role of connectivity. Transportation Research Part A: Policy and Practice, 81, 4-15. http://doi.org/10.1016/j.tra.2014.12.012

SOBRINO, N., y MONZON, A. (2014). The impact of the economic crisis and policy actions on GHG emissions from road transport in Spain. Energy Policy, 74, 486-498. http://doi. org/10.1016/j.enpol.2014.07.020

SOUTHWORTH, M. (2005). Designing the Walkable City. Journal of Urban Planning and Development, 131(4), 246-257. http://doi.org/10.1061/(ASCE)07339488(2005)131:4(246)

TRULLÉN, J. (dir.) (2014). Crisi econòmica, creixement de les desigualtats i transformacions socials. Informe general Enquesta de Condicions de vida i Hàbits de la Població de Catalunya, 2011. Bellaterra.

TRULlÉN, J., y GALLETTO, V. (2011). Per afrontar la crisi: la metròpoli de Barcelona. Barcelona.

URRY, J. (2000). Sociologies beyond Societies: Mobilities for the Twenty-First Century. Routledge.

ZAFRILLA, J. E., LÓPEZ, L. A., CADARSO, M. Á., y DEJUÁN, Ó. (2012). Fulfilling the Kyoto protocol in Spain: A matter of economic crisis or environmental policies? Energy Policy, 51, 708-719. http://doi.org/10.1016/j.enpol.2012.09.011 